\title{
Comparative X-ray Standing Wave Analysis of Metal-Phosphonate Multilayer Films of Dodecane and Porphyrin Molecular Square
}

\author{
Joseph A. Libera, ${ }^{\dagger}$ Richard W. Gurney, ${ }^{\ddagger} §$ Craig Schwartz, ${ }^{\ddagger}$ Hua Jin, ${ }^{\dagger}$ Tien-Lin Lee, \\ SonBinh T. Nguyen, ${ }^{*}$ Joseph T. Hupp, ${ }^{*}$ and Michael J. Bedzyk*,† \\ Department of Materials Science and Engineering and The Nanoscale Science and Engineering Center, \\ Northwestern University, Evanston, Illinois 60208, Department of Chemistry and The Nanoscale Science and \\ Engineering Center, Northwestern University, Evanston, Illinois 60208-3113, and European Synchrotron \\ Radiation Facility, BP 220, F-38043 Grenoble Cedex, France
}

Received: September 21, 2004; In Final Form: November 8, 2004

\begin{abstract}
The nanoscale structures of multilayer metal-phosphonate thin films prepared via a layer-by-layer assembly process using $\mathrm{Zr}^{4+}$ and 1,12-dodecanediylbis(phosphonic acid) (DDBPA) or porphyrin square bis(phosphonic acid) (PSBPA) were studied using specular X-ray reflectivity (XRR), X-ray fluorescence, and long-period $\mathrm{X}$-ray standing wave (XSW) analysis. The films were prepared in 1, 2, 3, 4, 6, and 8 layer series on both $\mathrm{Si}(001)$ substrates for XRR and on $18.6 \mathrm{~nm}$ period Si/Mo layered-synthetic microstructure X-ray mirrors for XSW. After functionalizing the $\mathrm{SiO}_{2}$ substrate surfaces with a monolayer film terminated with phosphonate groups, the organic multilayer films were assembled by alternating immersions in (a) aqueous solutions containing $\mathrm{Zr}^{4+}$ or $\mathrm{Hf}^{4+}$ (final metal layer only) cations and then (b) organic solvent solutions of $\mathrm{PO}_{3}-\mathrm{R}-$ $\mathrm{PO}_{3}{ }^{4-}$, where $\mathrm{R}$ was DDBPA or PSBPA spacer molecule. The $\mathrm{Hf}^{4+}$ cation served as the marker for the top surface of the films, whereas the $\mathrm{Zr}^{4+}$ cation was present in all other layers. The PSBPA also contained $\mathrm{Zn}$ and $\mathrm{Re}$ atoms at its midline which served as heavy-atom markers for each layer. The long-period XSW generated by the 0th- (total external reflection) through 4th-order Bragg diffraction conditions made it possible to examine the Fourier transforms of the fluorescent atom distributions over a much larger $q_{z}$ range in reciprocal space which permitted simultaneous analysis of $\mathrm{Hf}, \mathrm{Zn} / \mathrm{Re}$, and $\mathrm{Zr}$ atomic distributions.
\end{abstract}

\section{Introduction}

The design and evaluation of layer-by-layer assembled monoand multilayer films critically depends on knowledge of the asdeposited structural nature of the films. Herein we report a study using long period X-ray standing wave (XSW) analysis to determine the structural and chemical properties of two series of metal-phosphonate films based on 1,12-dodecanediylbis(phosphonic acid) (DDBPA) and porphyrin square bis(phosphonic acid) (PSBPA). Metal-phosphonate thin-film architectures owe their popularity to the ease of fabrication and the versatility in choice of both the metallic and phosphonate components. The layer-by-layer assembly process relies on the coordination chemistry of phosphonate-terminated molecules and the various transition metal ions.

Thin-films based on mono- and multilayer metal-phosphonate chemistry continue to attract attention in modern materials chemistry, primarily because the molecular structure of the phosphonate component can be easily modified to yield stable functional materials with tunable properties. ${ }^{1,2}$ Multifunctional,

* To whom correspondence should be addressed. Address: Northwestern University, Department of Materials Science and Engineering, 2220 Campus Drive, Evanston, IL 60208. Tel: (847) 491-3570. Fax: (847) 467-2269. E-mail: bedzyk@northwestern.edu.

$\dagger$ Department of Materials Science and Engineering and The Nanoscale Science and Engineering Center, Northwestern University.

$\doteqdot$ Department of Chemistry and The Nanoscale Science and Engineering Center, Northwestern University.

$\S$ Present Address: Department of Chemistry, Simmons College, Boston, MA 02130.

"European Synchrotron Radiation Facility. microporous thin-films with uniform thicknesses and a welldefined porous structure are desirable from a functional nanomaterials perspective. Microporous thin-film materials can function as molecular sieves,${ }^{2-4}$ frameworks for size-selective heterogeneous catalysis, ${ }^{5}$ chemical sensors, ${ }^{6,7}$ and in liquidjunction solar cells, ${ }^{8}$ when an appropriate chromophoric molecular framework is chosen. Pillared organic microporous films containing pores that are in the $10-20 \AA$ range were formed ${ }^{9}$ by cross-linking zirconium phosphate-like layers with several types of diphosphonic acids. Direct assembly of tetrameric porphyrin arrays has been studied by drop casting from toluene solutions of porphyrin square molecules based on Pt and Pd. ${ }^{10}$

Metal-phosphonate films based on $\mathrm{Zr}^{4+}$ and DDBPA have been thoroughly characterized, ${ }^{11,12}$ making this system a good standard sample in comparative structural studies. Putvinski and co-workers measured an experimental thickness of $15 \AA$ Alayer for a multilayer film series of $\mathrm{Zr}^{4+} / \mathrm{DDBPA}$ (expected thickness is $19.5 \AA$ /layer) deposited on Au substrates that had been primed with (8-mercaptooctyl)phosphonic acid. Schilling and coworkers $^{12}$ found that measured film thickness values were consistent with expectation when the phosphonate layering reaction was allowed to incubate for approximately $1-3$ days. In particular, a monolayer of DDBPA only gave a measured thickness of $13 \AA$ when a 2-h incubation time was used but reached $21 \AA$ when a 1-day incubation was used. As an example of the inherent variability of the layer thicknesses of metalphosphonate thin films, we cite Zeppenfeld et al., ${ }^{13}$ who prepared multilayers of $\mathrm{Hf}^{4+} / 1,10$-decanediylbis(phosphonic acid) (DBPA) on $\mathrm{Hf}^{4+}$-primed substrates. The preparative conditions for the 

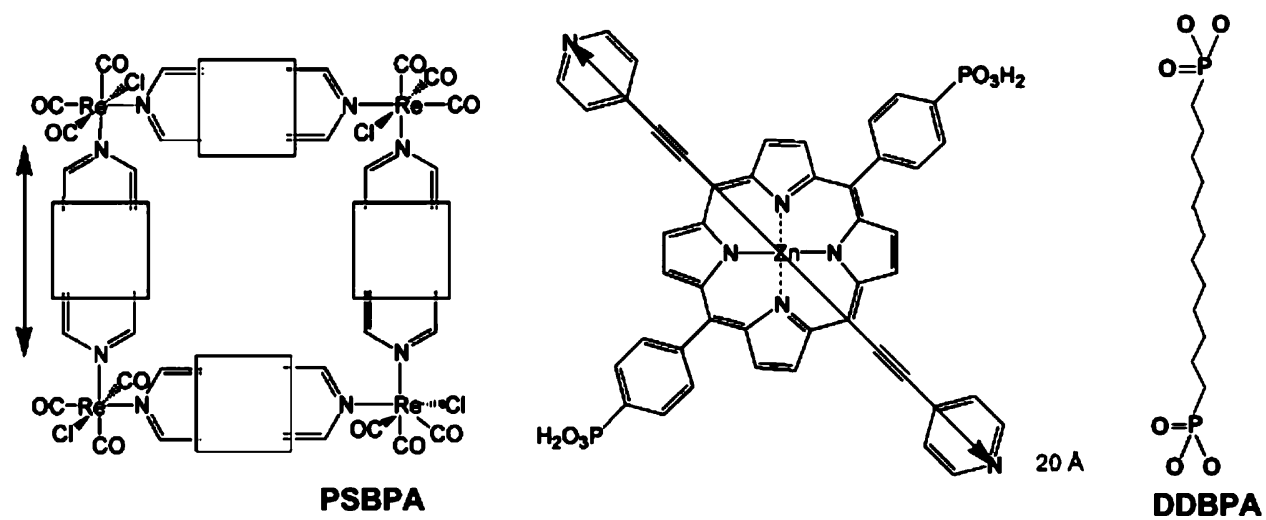

Figure 1. Molecular diagrams of 1,12-dodecanediylbis(phosphonic acid) (DDBPA) and porphyrin square bis(phosphonic acid) (PSBPA) phosphonate molecules. In the PSBPA molecule, the $\mathrm{Zn}$ and Re atoms form a plane that is expected to lie parallel to the sample surface. The four porphyrin constituents are expected to assemble in the films with the $\mathrm{PO}_{3}-\mathrm{PO}_{3}$ axes lying perpendicular to the plane of the Re atoms and thus perpendicular to the sample surface.

$\mathrm{Hf}^{4+}$ primer layer were varied as well as the deposition times for the subsequent $\mathrm{Hf}^{4+}$ /DBPA layers, which was kept at $\geq 4$ h. Ellipsometric thickness of the average per-layer thickness was found to vary from 14.8 to $20.7 \AA$, whereas the spacing in the bulk Hf-DBPA solid was reported to be $16.9 \AA$. This large variation in range was attributed to the packing density and conformation of the $\mathrm{Hf}$ deposited in the initial primer step which influences subsequent layer structure. We note that metal phosphonate films with more well-defined layer structures have been prepared using a combination of Langmuir-Blodgett techniques and layer-by-layer assembly. ${ }^{14}$

The major challenge in the design of functional thin-film materials is the elucidation of their structure and quality. This is particularly true in the formation of metal-phosphonate multilayer assemblies where the phosphonate spacer molecules have large asymmetric forms. In the present study, we use longperiod X-ray standing waves (XSWs) generated by total external reflection (TER) ${ }^{15,16}$ and Bragg diffraction from a layeredsynthetic-microstructure (LSM) ${ }^{17,18}$ to examine the heavy-atom profiles within the layer-by-layer assembled metal-phosphonate films that are grown on the $\mathrm{SiO}_{2}$ surface of the LSM. Within each film, we selectively generate an XSW with a period ranging from 20.9 to $4.5 \mathrm{~nm}$ by using the 0th- (TER) to 4th-order Bragg diffraction conditions from an underlying Si/Mo LSM which has an 18.6-nm $d$ spacing. Quantitative structural information is derived by comparing the measured heavy-atom fluorescence yield modulations to that predicted by a dynamical X-ray scattering theory. In addition to measuring the individual heavy atom $\rho(\mathrm{z})$ profiles with XSW, we also measure the coverage of each heavy element by X-ray fluorescence. We also measure the overall electron density profile of the films with specular $\mathrm{X}$-ray reflectivity (XRR) performed on a series of related thinfilm samples that were deposited on plain Si substrates with a $\mathrm{SiO}_{2}$ surface layer, but without the intervening LSM.

To best utilize XSW techniques, an arrangement of several heavy atoms that mark different structural features was chosen. In a previous XSW study ${ }^{19}$ of similar metal-phosphonate films, we found that the best metal layer arrangement is to use $\mathrm{Zr}^{4+}$ for all metal atom layers except the last (top), for which $\mathrm{Hf}^{4+}$ was used. In addition to the structural metal atoms $\mathrm{Zr}^{4+}$ and $\mathrm{Hf}^{4+}$, the PSBPA molecule that we used to form the nanoporous metal-phosphonate films in this study also contains $\mathrm{Zn}$ and Re atoms that are expected to be located at the midline of the phosphonate sandwich. This design feature nominally provides a metal atom distribution that should be identical to the $\mathrm{Zr}$ atom distribution shifted vertically by one-half of a single layer thickness.

\section{Experimental Section}

Substrate Fabrication. The metal-phosphonate films were grown on $\mathrm{SiO}_{x}$ surfaces. The $\mathrm{SiO}_{x}$ surfaces were atop of Si(001) substrates or atop of the final sputter-deposited Si layer of Si/Mo LSM mirrors that were deposited on Si(001) substrates (37.5-mm long by $12.5-\mathrm{mm}$ wide by $2.5-\mathrm{mm}$ thick). The surface roughness of these pre-polished $\mathrm{Si}(001)$ wafers was $2-5 \AA \mathrm{rms}$. The Si/Mo LSMs were prepared as a single batch by the Optics Group of the Advanced Photon Source at Argonne National Laboratory using DC magnetron sputtering at an Ar pressure of 2.3 mTorr. Each LSM had $N=20 \mathrm{Si} /$ Mo bilayers deposited. The XRR analysis of these LSMs, which will be described below, determined that the bilayer thickness (period) was $d=$ $18.6 \mathrm{~nm}$ and the Si:Mo thickness ratio was 6.14. The terminating surface of the LSM was the final Si layer. The actual thickness of this top Si layer was slightly larger than the other Si layers due to the $\mathrm{SiO}_{x}$ native oxide growth.

Film Preparation. All substrates in this study received an identical primer treatment prior to subsequent layer deposition. The primer chemistry was based on the work of Horne et al., ${ }^{20}$ with slight modifications. In the first step, the substrates were placed in piranha solution (2:1, sulfuric acid: hydrogen peroxide) to remove organic contaminants from the surface and then rinsed with ultrapure Millipore water and dried under a stream of $\mathrm{N}_{2}$. (Caution! Piranha solution can react violently with organics.) Immediately afterward the substrates were immersed in 2-M HCl for $5 \mathrm{~min}$, rinsed with ultrapure-water, dried under a stream of dry $\mathrm{N}_{2}$, and oven-dried at $80{ }^{\circ} \mathrm{C}$ for 15 min. The substrates were placed in an $80{ }^{\circ} \mathrm{C}$ solution of anhydrous octanol and (3-aminopropyl)trimethoxysilane (APTMS) (100:1 v:v) for $10 \mathrm{~min}$; followed by rinsing with hexanes and ultrapure-water, drying under a stream of dry $\mathrm{N}_{2}$, and drying in an oven at $80^{\circ} \mathrm{C}$ for $30 \mathrm{~min}$. Phosphorylation was followed by placement into a mixture of $0.1-\mathrm{M} \mathrm{POCl}_{3}$ and 0.1-M 2,4,6-collidine in anhydrous acetonitrile (ACN), for $1 \mathrm{~h}$. The samples were then heated in warm, dry ACN for 15 min, followed by rinses with ACN and ultrapure water. The samples were then dried under a stream of dry $\mathrm{N}_{2}$ and then placed in an aqueous solution of $5-\mathrm{mM} \mathrm{Z \textrm {ZOCl } _ { 2 }} \cdot 8 \mathrm{H}_{2} \mathrm{O}$ for $2 \mathrm{~h}$, rinsed with ultrapure-water, and dried under a stream of dry $\mathrm{N}_{2}$.

In Figures 1 and 2, we show the schematic structure of the phosphonate molecules and layer-by-layer films, respectively. We adopt a sample naming convention defined in Figure 2. As examples, the 1-layer DDBPA film on an XSW substrate is denoted DD1X and the 8-layer PSBPA film on a reflectivity 


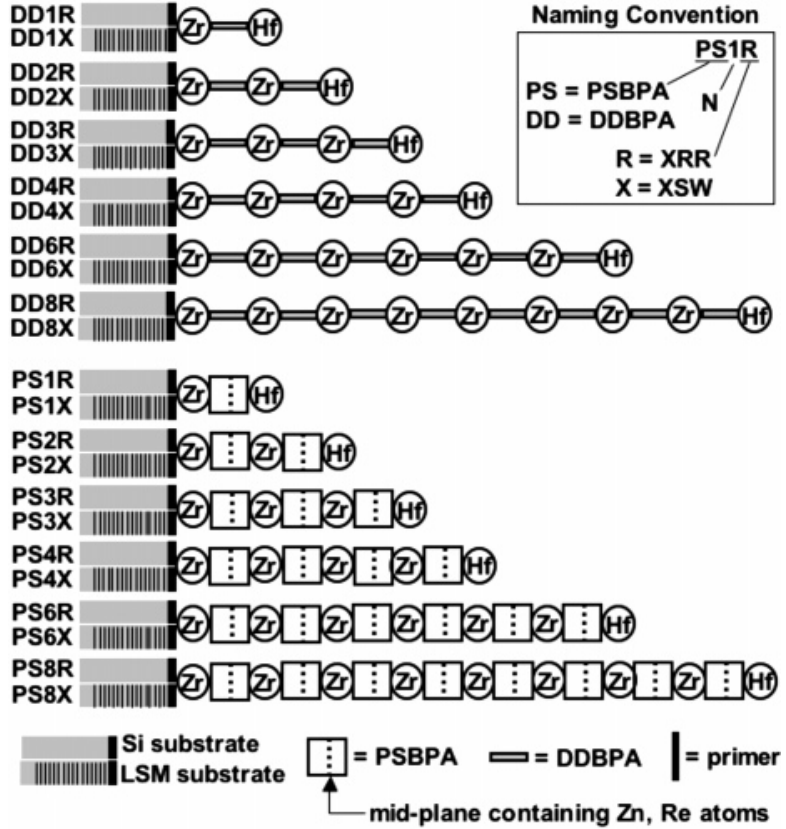

Figure 2. Schematic diagram for the preparation of the layered sample films. Circles indicate immersion of the samples into aqueous solutions of the indicated atom. The narrow shaded rectangle indicates immersion into an ethanolic solution of DDPBA. The square indicates immersion into a solution of PSBPA in dimethyl sulfoxide. Each pair of substrates (for example DD1X and DD1R) on the left received an identical preparation as indicated by the layering sequence on the right.

substrate is denoted PS8R. Figure 1 shows the molecular structure of DDPBA used in the DDnR and DDnX series and the PSBPA molecule used for PS $n \mathrm{R}$ and PSnX series $(n=1$, 2, 3, 4, 6, and 8). In Figure 2, we show the sequence of assembly for each sample where each circle or rectangle represents a single immersion step into an appropriate solution as described below: (1) DDBPA 1.0-mM 1,12-dodecanediylbis(phosphonic acid) in $60 \%$ ethanolic solution for 2 h. (2) PSBPA: 0.0125 $\mathrm{mM}$ solution of porphyrin square bis(phosphonic acid) in DMSO for $2 \mathrm{~h}$. (3) $\mathrm{Zr}$ : $5-\mathrm{mM} \mathrm{ZrOCl}_{2} \cdot 8 \mathrm{H}_{2} \mathrm{O}$ for $30 \mathrm{~min}$. (4) $\mathrm{Hf}$ : $5-\mathrm{mM}$ $\mathrm{HfOCl}_{2} \cdot 8 \mathrm{H}_{2} \mathrm{O}$ for $30 \mathrm{~min}$. Full details of the synthesis and characterization of the DDBPA and PSBPA molecules are given elsewhere. $^{21}$

XSW Experiments. Long-period X-ray standing wave (XSW) analysis is generally used for measuring element-specific atomic distribution profiles $\rho(\mathrm{z})$ in a surface overlayer structure. In the present case, the XSW is generated by total external reflection (TER) ${ }^{16,22}$ and Bragg diffraction from a periodic layered-synthetic-microstructure (LSM). ${ }^{17,18}$ The XSW, which is produced by the interference between the incident and reflected X-ray plane waves, has planes of equal intensity (nodes and antinodes) that are parallel to the LSM interfaces. (We will choose the $z$ direction to be perpendicular to this set of parallel interfaces.) In the vacuum (or air) region above the reflecting surface, the XSW period is $D=\lambda / 2 \sin \theta=2 \pi / q$. Even though the X-ray wavelength, $\lambda$, is on the order of angstroms, the XSW period can be very much longer (varying with angle from a couple of microns to a couple of nanometers), since TER and Bragg diffraction from an LSM occur at very small incident angles, $\theta$, or more generally at small values of scattering vector, $q$. The phase $(v)$ of the XSW shifts inward by $180^{\circ}$ as the incident angle $(\theta)$ is scanned through the region of TER or across a Bragg reflection. This induces a modulation in the X-ray fluorescence yield from a given atom that is characteristic of the spatial distribution profile $\rho(z)$ for that atomic species.
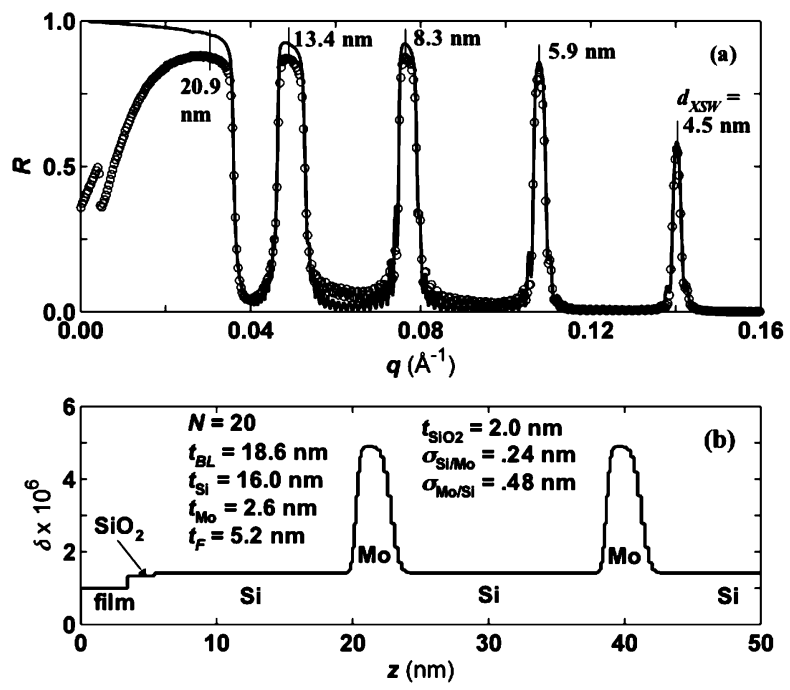

Figure 3. (a) Typical measured X-ray reflectivity data (filled circles) taken simultaneously during the XSW scan along with a dynamical theory fit to the data (solid line) for sample PS3X. (b) The electron density profile used to model the LSM + film shown by plotting the real part of the index of refraction, $\delta$. Only the first 2 of $20 \mathrm{LSM} \mathrm{Si} /$ Mo bilayers are shown.

The measured atomic fluorescence yield $Y(\theta)$ at any incident angle $\theta$ is the integral of the product of the unknown atomic distribution $\rho(z)$ and the calculated $E$-field intensity $I(\theta, z)$. For cases in which the sample films are very thin (e.g., less than 1 $\mathrm{nm}$ ) and of much lower density than the substrate, the film will only have a small effect on the $E$-field intensity within the film. This is especially true for angles greater than the critical angle for the film. However, in this study, we include samples with an overall film thickness of $\sim 16 \mathrm{~nm}$ which have a strong effect on the $E$-field intensity in the film at very small angles so we must include the films in the dynamical model used to compute the $E$-field.

In previous long-period XSW studies using LSMs, ${ }^{15,22}$ the LSM period was typically in the range of 2.5 to $8 \mathrm{~nm}$ and the XSW analysis only made use of the 0th (TER) and 1st order Bragg peaks. The higher-order Bragg peaks were typically too weak $\left(R_{\text {peak }}<15 \%\right)$ to generate an XSW with sufficient fringe visibility. In this study, we use an LSM with a much larger $d$ spacing to produce a variable period XSW over several orders of Bragg diffraction peaks. In Figure 3a, we show a typical measured and calculated X-ray reflectivity from sample PS3X. Figure $3 \mathrm{a}$ also lists the XSW standing wave period $(D)$ in the vacuum at each Bragg peak position. Note that at very small incident angles refraction causes an upward shift of the external Bragg angle and also causes the XSW period outside the LSM to be significantly smaller than the period $\left(d_{\mathrm{m}}\right)$ inside the LSM. This ability to generate a variable period XSW over a large range from 4 to $21 \mathrm{~nm}$ allows us to measure atomic distributions over these same length scales. In essence, the measured modulations in the fluorescence yield from an overlayer atom leads to a measure of the Fourier transform $F(q)$ of the distribution $\rho(z)$ of that atom.

XSW measurements of the DDnX and PSnX series were performed at the European Synchrotron Radiation Facility (ESRF) ID32 beamline using $E_{\gamma}=18.50 \mathrm{keV}(\lambda=0.670 \AA)$ incident X-rays to excite $\mathrm{Zr} \mathrm{K} \alpha, \mathrm{Zn} \mathrm{K} \alpha, \operatorname{Re} \mathrm{L} \alpha$, and $\mathrm{Hf} \mathrm{L} \alpha$ $\mathrm{X}$-ray fluorescence. The incident beam was conditioned by a double-crystal Si (111) monochromator followed by a 0.02 mm-high by $1.0-\mathrm{mm}$-wide slit. Knife edge scans measured the actual vertical size fwhm to be $0.015 \mathrm{~mm}$. The reflectivity and fluorescence data were simultaneously collected in a $\theta-2 \theta$ 


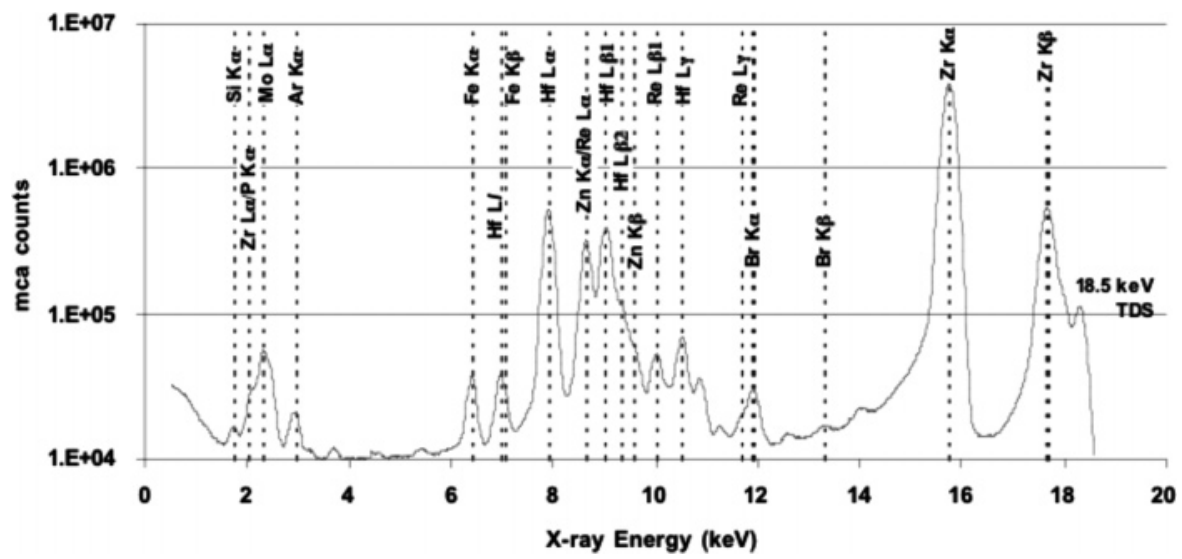

Figure 4. Typical PSnX series X-ray fluorescence spectrum showing the angle-integrated spectrum from the XSW scan of sample PS8X. All labeled lines (except Fe and $\mathrm{Br}$ lines) give characteristic film or LSM XSW fluorescence yield curves. The DDBPA series samples give similar spectra with the $\mathrm{Zn}$ and Re lines absent.

scan covering the range of TER through the first four Bragg peaks of the LSM mirror. The high photon flux $\left(3 \times 10^{10} \mathrm{p} / \mathrm{s}\right)$ at the ID32 beamline permitted fast high-resolution scans to be taken (600 steps from $q=0.0$ to $0.2 \AA^{-1}$ with a 5 -s counting time for a total time of $50 \mathrm{~min}$ ). Multichannel-analyzer (MCA) $\mathrm{X}$-ray fluorescence spectra were collected at each angle step of the scan using a high count-rate RONTEC Si-drift diode detector. A $\sin \theta$ geometrical X-ray beam footprint correction was applied to the dead-time- and efficiency-corrected fluorescence data. Finally, the fluorescence yields were normalized to unity at an angular position where the reflectivity was zero.

XRR Experiments. X-ray reflectivity (XRR) was performed at the Northwestern University X-ray Facility using $\mathrm{Cu} \mathrm{K \alpha}(8.04$ $\mathrm{keV}) \mathrm{X}$-rays from a rotating anode vertical line source coupled to a parabolic, graded $d$ spacing, collimating, multilayer mirror, followed by a 2 -circle diffractometer. The beam size was 0.10 mm-wide by 10 -mm-high. The instrumental resolution was $\Delta q$ $=5 \times 10^{-3} \AA^{-1}$. The XRR data from the NaI detector was dead-time corrected and background subtracted.

XRF Coverage Measurement. The absolute coverage of each of the heavy atoms within each film was measured by an $\mathrm{X}$-ray fluorescence (XRF) comparison to an arsenic-implanted standard that had an effective coverage of $1.04 \times 10^{15} \mathrm{As} / \mathrm{cm}^{2}$ that was calibrated by Rutherford backscattering (RBS). MCA spectra were collected from the standard and all samples using identical incident beam and fluorescence detector collection geometry. At $E_{\gamma}=18.5 \mathrm{keV}$, the sensitivity factors ${ }^{23}$ for $\mathrm{Hf}$ $\mathrm{L} \alpha, \mathrm{Zn} \mathrm{K} \alpha, \operatorname{Re} \mathrm{L} \alpha$, and $\mathrm{Zr} \mathrm{K} \alpha$ relative to $\mathrm{As} \mathrm{K} \alpha$ are 0.663, $0.623,0.939$, and 2.33 , respectively.

\section{Results}

XSW Measurements. The sample reflectivity for each film of the DDnX and PSnX series was modeled from reflectivity data collected simultaneously during the XSW scans by applying Parratt's recursion formulation ${ }^{24}$ to a layered model consisting of a series of layers (slabs), each layer having a constant index of refraction $\left(n_{j}=1-\delta_{j}-i \beta_{j}\right)$, thickness $\left(t_{j}\right)$, and interfacial width $\left(\sigma_{j}\right)$. The LSM portion of the model included a detailed treatment of the $\mathrm{Si} / \mathrm{Mo}$ interfacial structure and accounted for bilayer thickness variation. An example of the measured and calculated reflectivity is shown in Figure 3a for sample PS3X. The electron density profile is shown in Figure $3 \mathrm{~b}$ for the first two periods of the LSM. Full details of the LSM reflectivity modeling were reported previously. ${ }^{19}$ The metal-phosphonate multilayer film was included by adding a single layer to the model.
An X-ray fluorescence emission spectrum typical of PSnX series samples is shown in Figure 4 for sample PS8X. The displayed spectrum is the angle-integrated spectrum (summation of 601 individual X-ray spectra) from the XSW scan. With the exception of the Fe and $\mathrm{Br}$ peaks, all labeled X-ray lines were analyzed and showed fluorescence yield curves that were characteristic of atoms in the LSM or sample films. Since we are interested here in the properties of the metal-phosphonate films, we present only the XSW yield data for those metals found in the films, namely, Hf, Zn, Re, and Zr. The X-ray spectra for samples in the $\mathrm{DD} n \mathrm{X}$ series are similar to those for the $\mathrm{PS} n \mathrm{X}$ series after the $\mathrm{Zn}$ and Re lines are removed.

To calculate the XSW yield, we first calculate the $E$-field intensity $I(\theta, z)$ at any point in the sample film layer using an extension of Parratt's recursion formulation. ${ }^{25,26}$ The fluorescence yield is then calculated according to the integral $Y(\theta)=$ $\int \rho(z) I(\theta, z) \mathrm{d} z$ for various models, $\rho(z)$, of the unknown atomic distribution of the metal atom layers. The measured and calculated $\mathrm{Hf}$ and $\mathrm{Zr}$ yields for all samples in the $\mathrm{DD} n \mathrm{X}$ series are shown in Figures 5 and 6, respectively. The models for the $\chi^{2}$ fits used a Gaussian profile, $\rho_{\mathrm{G}}(z)$, for the $\mathrm{Hf}$ and a rectangular profile, $\rho_{\mathrm{R}}(z)$, for the $\mathrm{Zr}$ as shown in the insets in Figures 5 and 6 . The free fitting parameters for the Hf distribution were the mean position $z_{0}$ and width $\sigma$ of the Gaussian distribution. The free fitting parameters for the $\mathrm{Zr}$ distribution were the midheight, $z_{0}$, and width, $\Delta z$, of the rectangular distribution. Also included in each model was an extended uniform distribution, $\rho_{\mathrm{E}}(z)$, to account for atoms not contained in the assumed profiles. The final distribution was $\rho(z)=C \rho_{\mathrm{M}}(z)+(1-C) \rho_{\mathrm{E}}(z)$, where $C$ is the partition fraction and $\rho_{\mathrm{M}}(z)$ is either the Gaussian or rectangular profile. A fixed value of $C=0.85$ was used for all of the distributions in this study. The effect of including an extended uniform distribution is to allow for a component of fluorescence yield that is proportional to $1+R$. Referring to Figure 2, the PSnX films were assembled in the same manner as the DDnX films, except using PSBPA in place of DDBPA. Consequently, we expect the same structure with the addition of $\mathrm{Zn}$ and $\mathrm{Re}$. The $\mathrm{Zn}$ and $\mathrm{Re}$ are expected to be located at the mid-plane of the ideally configured porphyrin square molecule or equidistant from adjacent $\mathrm{Zr}$ or $\mathrm{Hf}$ metal atom layers. The strong fluorescence lines from $\mathrm{Zn}$ and $\mathrm{Re}$ are the $\mathrm{Zn} \mathrm{K \alpha}$ and Re L $\alpha$ X-ray lines which are separated by only $20 \mathrm{eV}$ and thus were analyzed together as a single fluorescence. Although we also see $\mathrm{ZnK} \beta$ and $\operatorname{Re} \mathrm{L} \alpha$ peaks in our spectra, these peaks were too weak to be analyzed over the full angular range. At low angles where the fluorescence intensity is highest, fluores- 


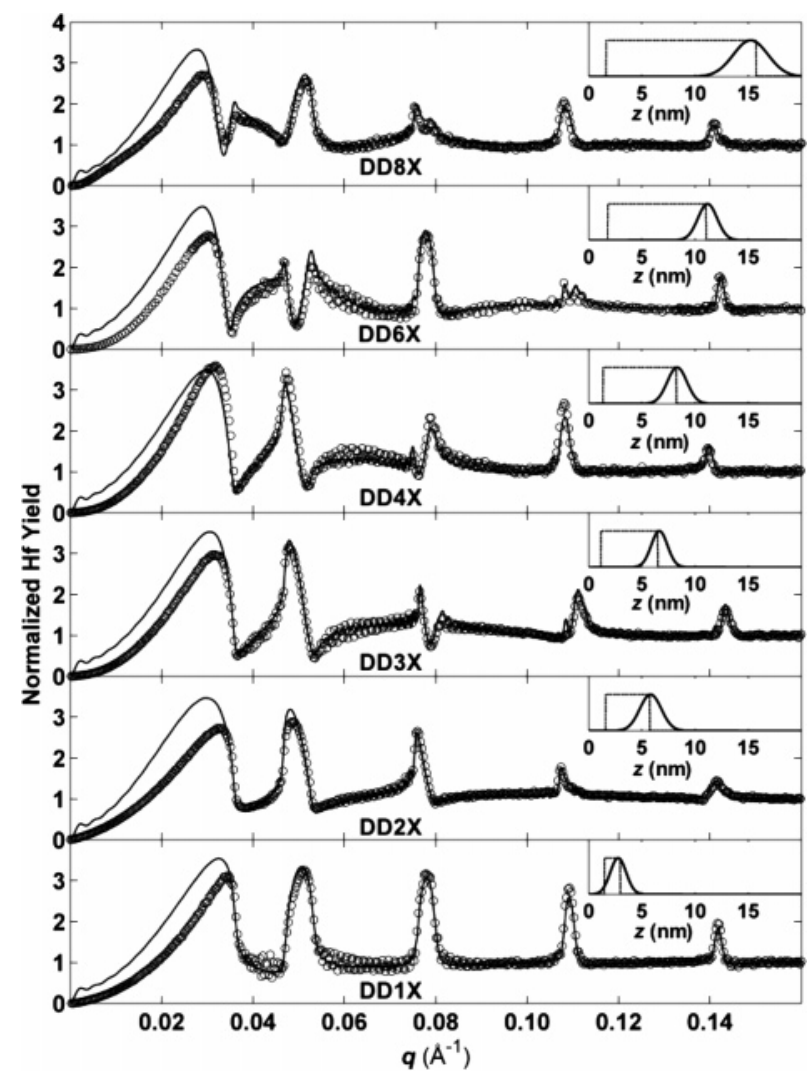

Figure 5. DD $n \mathrm{X}$ series $\mathrm{XSW}$ results showing the measured $\mathrm{X}$-ray fluorescence yields for Hf (open circles) and $\chi^{2}$ fits (solid lines). For each sample, the inset shows the atomic distribution used for $\mathrm{Hf}$ (solid line) along with the atomic distribution used for $\mathrm{Zr}$ (dashed line). The atomic distributions are scaled vertically for clarity and are not proportional to the atomic coverages.

cence yields from the $\mathrm{Zn} \mathrm{K} \alpha$ and $\mathrm{Re} \mathrm{L} \alpha$ peaks (results not shown) were very similar to the $\mathrm{Zn}+$ Re yield, indicating that both atoms were equally represented by the combined $\mathrm{Zn} \mathrm{K \alpha}$ $+\operatorname{Re} L \alpha$ peak.

The measured and calculated $\mathrm{Hf}, \mathrm{Zn} / \mathrm{Re}$, and $\mathrm{Zr}$ yields are shown for each sample in the PSnX series in Figures 7-9, respectively. The theoretical fits were obtained using an identical procedure as for the $\mathrm{DD} n \mathrm{X}$ series using a Gaussian atomic distribution for $\mathrm{Hf}$ and rectangular distributions for $\mathrm{Zn}+\mathrm{Re}$ and $\mathrm{Zr}$. However, for the rectangular distributions for $\mathrm{Zn}+\mathrm{Re}$ and $\mathrm{Zr}$, we observed a coupling between the mid-height, $z_{0}$, and width, $\Delta z$, in several of the PSnX series samples that we did not see in the DDnX series. We obtained much better results by fixing $\Delta z$ using a constraint based on the Hf fit result. For each sample in the series, the width of the rectangular distribution for both the $\mathrm{Zr}$ and $\mathrm{Zn}+\mathrm{Re}$ was set to $\Delta z=z_{0, \mathrm{Hf}}-1.3$ $\mathrm{nm}$. This relation was deduced from the values observed when $\Delta z$ was left to be a free fitting parameter in those samples in the PSnX series that did not show the coupling between parameters. All of the model parameters for the atomic distributions of $\mathrm{Hf}, \mathrm{Zn}+\mathrm{Re}$, and $\mathrm{Zr}$ are summarized in Table 1 and are plotted in Figure 10. In Figure 10, we have plotted the mean position of the Gaussian profile and the bottom, midheight, and top of the rectangular profiles where the top and bottom are calculated from the mid-height and width. The XRF coverage results for the PSBPA series samples are given in Table 1 and plotted in Figure 11. The atomic coverages of the $\mathrm{DD} n \mathrm{R}, \mathrm{DD} n \mathrm{X}$, and $\mathrm{PS} n \mathrm{R}$ series samples were measured using $18.5 \mathrm{keV} \mathrm{X}$-rays at the 5BM-D beamline at the Advanced Photon Source, Argonne National Laboratory and are listed in Tables 1 and 2 and plotted in Figure 11.

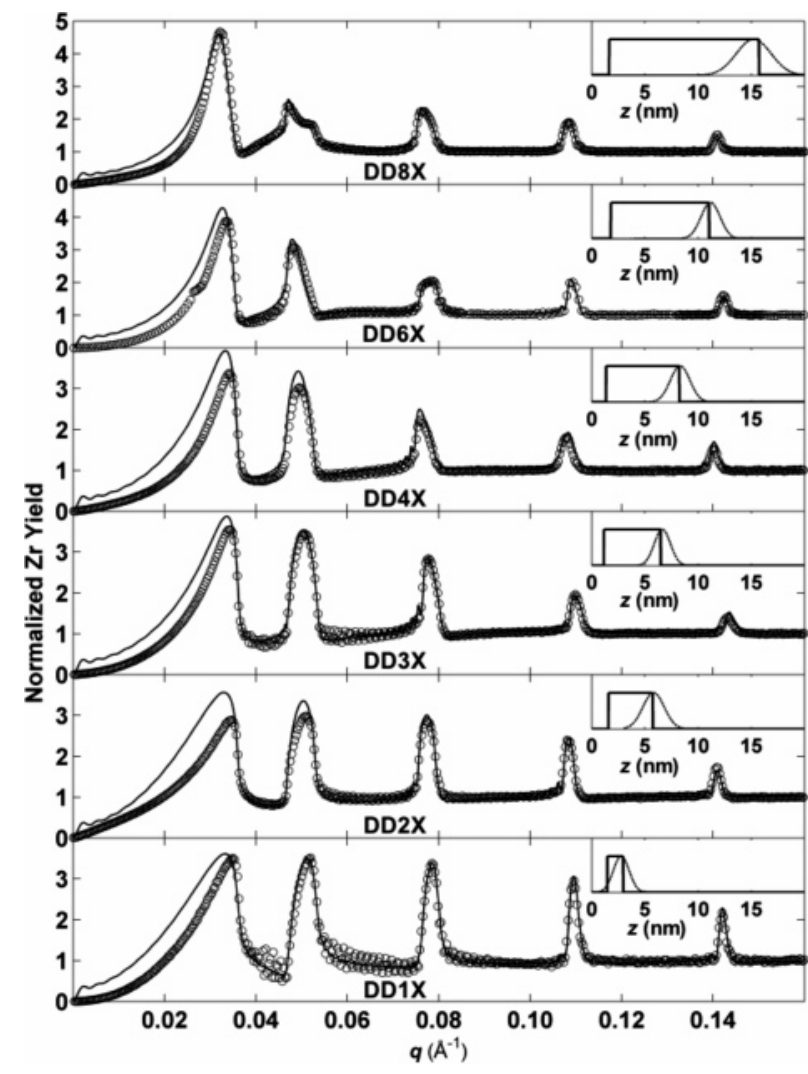

Figure 6. $\mathrm{DD} n \mathrm{X}$ series $\mathrm{XSW}$ results showing the measured $\mathrm{X}$-ray fluorescence yields for $\mathrm{Zr}$ (open circles) and $\chi^{2}$ fits (solid lines). For each sample, the inset shows the atomic distribution used for $\mathrm{Zr}$ (solid line) along with the atomic distribution used for Hf (dashed line). The atomic distributions are scaled vertically for clarity and are not proportional to the atomic coverages.

XRR Measurements. The DD $n \mathrm{R}$ and PSnR sample series were prepared identically to series DD $n \mathrm{X}$ and $\mathrm{PS} n \mathrm{X}$, respectively, but on $\mathrm{Si}(001)$ substrates in order to provide suitable samples for XRR measurement. The experimental reflectivity data were fit by using the same dynamical scattering theory based on Parratt's recursive formulation ${ }^{22}$ as was used for modeling the LSMs of this study. A Nevot-Croce graded-layer treatment of the interfaces was required due to the large roughness. The layered model consisted of layers for the $\mathrm{Si}$ substrate, $\mathrm{SiO}_{2}$ surface oxide, and sample film with 10 additional layers to approximate the error function profile for the NevotCroce treatment of each interface. The sample film was treated as a single layer of uniform electron density. The fit parameters were the electron density of the film, film thickness, surface roughness, and interface roughness. Figure 12 shows the measured and calculated X-ray reflectivity for DDnR series samples with the inset showing the electron density profile for sample DD8R. Not shown are similar fits for the PSnR series. The XRR thicknesses, electron density and interface roughness are listed in Table 2 along with the XRF determined Hf, Zr, and $\mathrm{Zn}+\mathrm{Re}$ coverage for the $\mathrm{DD} n \mathrm{R}$ and $\mathrm{PS} n \mathrm{R}$ series. In Figure 13, the XRR determined thicknesses with $\chi^{2}$ fits are plotted for the $\mathrm{DD} n \mathrm{R}$ and $\mathrm{PS} n \mathrm{R}$ series as well as the Hf mean position, $z_{0, \mathrm{Hf}}$, for the companion $\mathrm{DD} n \mathrm{X}$ and PSnX series.

\section{Discussion}

We now use the foregoing analyses to discuss various aspects of the sample film structure. Our XSW analysis provides the atomic distribution profiles for each heavy atom contained within the sample films, and the XRF analysis provides the 


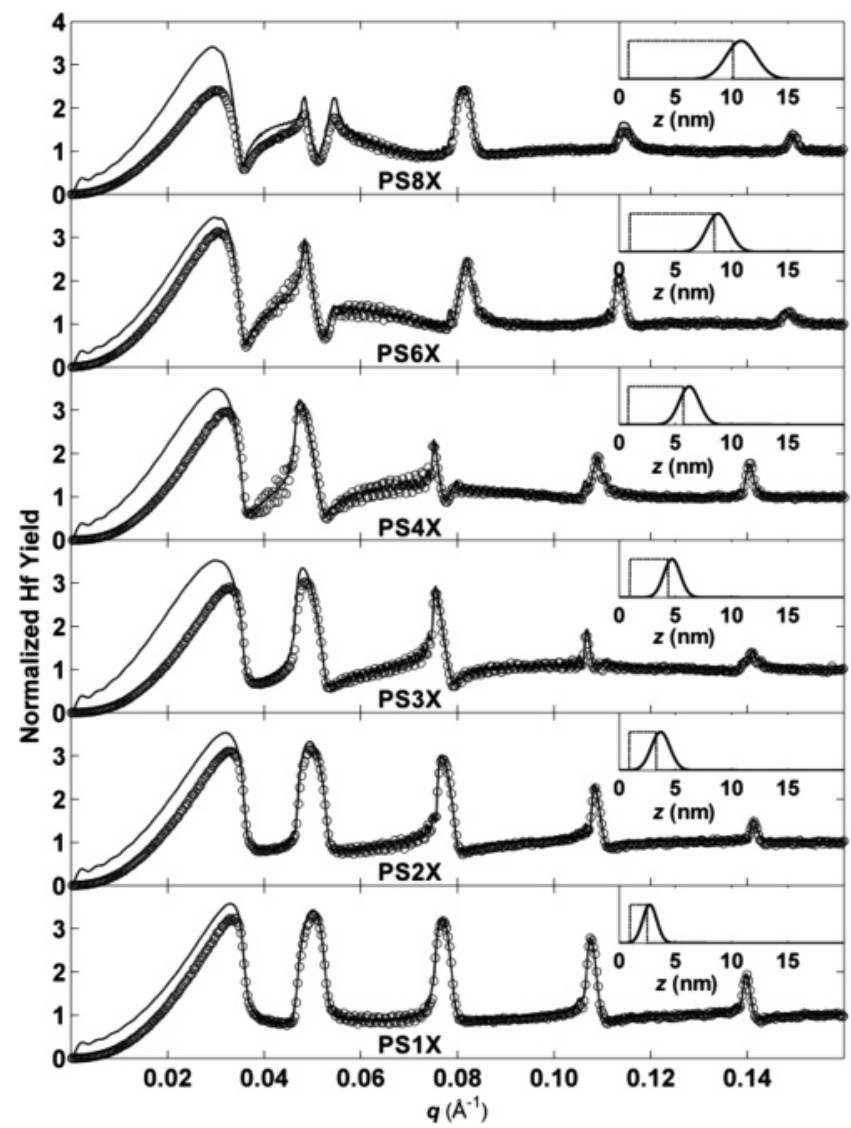

Figure 7. $\mathrm{PS} n \mathrm{X}$ series $\mathrm{XSW}$ results showing the measured $\mathrm{X}$-ray fluorescence yields for Hf (open circles) and $\chi^{2}$ fits (solid lines). For each sample, the inset shows the atomic distribution used for $\mathrm{Hf}$ (solid line) along with the atomic distribution used for $\mathrm{Zr}$ (dashed line). The atomic distributions are scaled vertically for clarity and are not proportional to the atomic coverages.

projected area density of each heavy atom. We can compare the atomic profiles and number density to the expected structure based on chemistry principles to check our assumption. The evolution of roughness as the number of layers increases gives an indication of the uniformity of tilt conformations. The film thickness as determined by XRR can be compared to the XSW measured mean position of the Hf top metal layer. Finally, we can compare the XRR determined electron density to that which is implied by the atomic coverages. The latter is particularly effective in the PSnX series for which metal atom coverages for each component in the system should give us the exact electron density in principle.

The DDBPA layer series (DDnX and DDnR) were chosen to provide a control with previous published structure data to compare to the previously unreported layer series based on the porphyrin square. The XSW determined structural properties of the DDnX series are shown Figure 10a, where we have plotted the Hf mean position, $z_{0, H f}$, together with the position of the top $\left(z_{\text {top }}=z_{0}+\Delta z / 2\right)$, middle $\left(z_{0}\right)$ and bottom $\left(z_{\text {bot }}=z_{0}\right.$ $-\Delta z / 2)$ of the rectangular profiles used to model the Zr. Also shown are $\chi^{2}$ fits of each parameter. The $\chi^{2}$ fit of the Hf mean position gives $z_{0, \mathrm{Hf}}=1.69 \mathrm{~N}+1.58(\mathrm{~nm})$. The per-layer increase in thickness of $1.69 \mathrm{~nm}$ is in good agreement with previously published values for this same system. ${ }^{12}$ The offset value of 1.58 represents the expected $\mathrm{Hf}$ mean position if it were used in place of $\mathrm{Zr}$ at the primer step. This value is higher than expected based on the length of a single APTMS/DDBPA/Zr moiety that was deposited as the primer layer and may be due to polymerization of the APTMS. The larger than expected

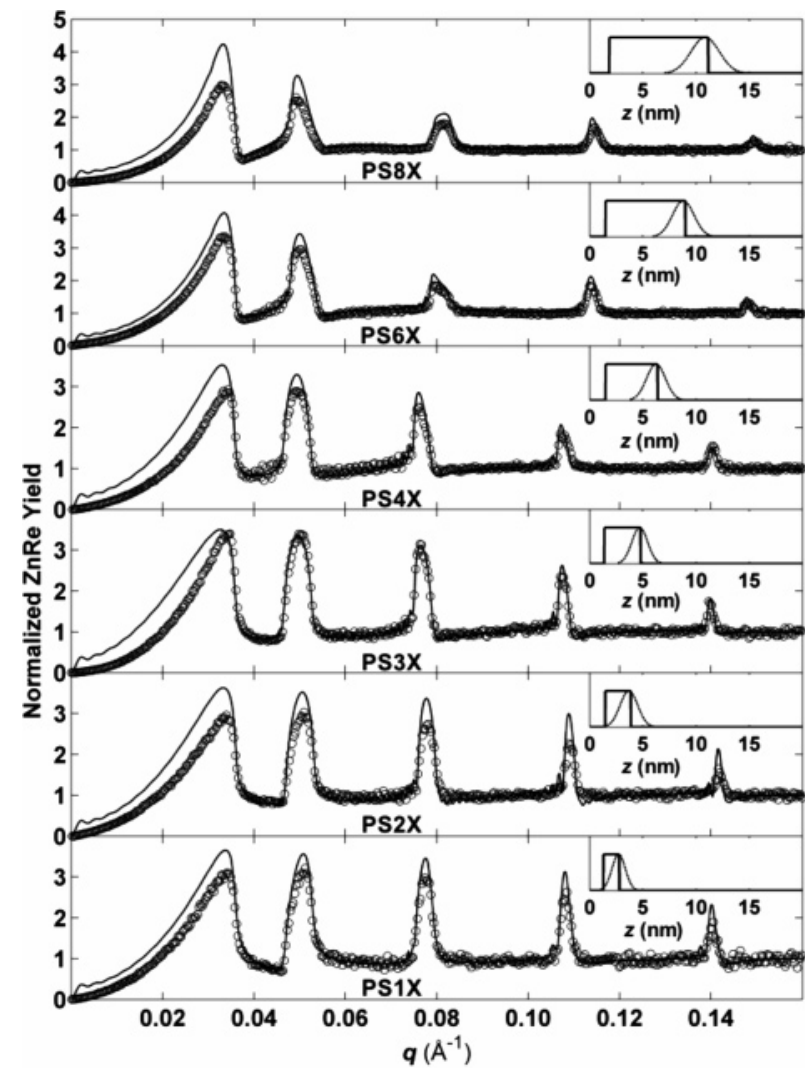

Figure 8. $\mathrm{PS} n \mathrm{X}$ series $\mathrm{XSW}$ results showing the measured $\mathrm{X}$-ray fluorescence yields for $\mathrm{Zn}+\operatorname{Re}$ (open circles) and $\chi^{2}$ fits (solid lines). For each sample, the inset shows the atomic distribution used for $\mathrm{Zn}$ $+\operatorname{Re}$ (solid line) along with the atomic distribution used for $\mathrm{Hf}$ (dashed line). The atomic distributions are scaled vertically for clarity and are not proportional to the atomic coverages.

offset is present in each of the four series in this study. For each sample in the $\mathrm{DD} n \mathrm{X}$ series, the top of the $\mathrm{Zr}$ atomic distribution is found to be very close to the Hf mean position. The deviations of the Hf mean position from the linear trend are closely followed by the top of the $\mathrm{Zr}$ rectangular profile. This is compelling evidence that these deviations represent true variation in sample structure. The average value of the bottom of the $\mathrm{Zr}$ atomic profiles is $1.49 \mathrm{~nm}$, which is close to the offset value of $1.58 \mathrm{~nm}$ of the Hf mean position.

Although the overall trends indicated by the Hf mean position and $\mathrm{Zr}$ rectangular profile parameters are in excellent agreement with the expected layer structure, individual samples in the series exhibit significant variation from the trends. Let us consider the simplest case of the one layer sample, DD1X. From Table 1, we see that the mean position of the Hf layer is $z_{0}=2.8 \mathrm{~nm}$ compared to $z_{0}=2.1 \mathrm{~nm}$ for the middle of the $\mathrm{Zr}$ distribution giving a separation between the $\mathrm{Hf}$ and $\mathrm{Zr}$ layers of $0.7 \mathrm{~nm}$ which is less than half of the per-layer thickness of $1.69 \mathrm{~nm}$. The largest deviation from the trend is sample DD2X whose Hf mean position is $5.81 \mathrm{~nm}$. From this value, we calculate a per-layer thickness of $(5.81-1.58) / 2=2.12$ $\mathrm{nm}$ that suggests that the DDBPA molecules are nearly perpendicular to the surface. This in turn implies a higher packing density in sample DD2X which is supported by the higher than average $\mathrm{Zr}$ and $\mathrm{Hf}$ atomic coverage for this sample (see Table 1 and Figure 11). Our general observation is that, although the overall trends suggested by both the $\mathrm{Hf}$ and $\mathrm{Zr}$ atomic profiles are in excellent agreement with the expected structure, there is significant variation of structure in individual samples. 
TABLE 1: XSW and XRF Results for the DDnX and PSnX Series Films

\begin{tabular}{|c|c|c|c|c|c|c|c|c|c|}
\hline \multirow[b]{2}{*}{ sample } & \multicolumn{3}{|c|}{ Hf } & \multicolumn{3}{|c|}{$\mathrm{Zr}$} & \multicolumn{3}{|c|}{$\mathrm{Zn}+\mathrm{Re}$} \\
\hline & $\overline{z_{0}{ }^{a}(\mathrm{~nm})}$ & $\sigma^{a}(\mathrm{~nm})$ & $\overline{\Theta^{c}\left(\mathrm{~nm}^{-2}\right)}$ & $\overline{z_{0}^{b}(\mathrm{~nm})}$ & $\Delta z^{b}(\mathrm{~nm})$ & $\Theta^{c}\left(\mathrm{~nm}^{-2}\right)$ & $\overline{z_{0}^{b}(\mathrm{~nm})}$ & $\Delta z^{b}(\mathrm{~nm})$ & $\Theta^{c}\left(\mathrm{~nm}^{-2}\right)$ \\
\hline DD1X & $2.76(3)$ & $1.05(4)$ & $5.7(6)$ & $2.20(2)$ & $1.50(1)$ & $4.3(4)$ & & & \\
\hline DD2X & $5.81(3)$ & $1.42(2)$ & $7.7(12)$ & $3.66(3)$ & $4.16(5)$ & $14(2)$ & & & \\
\hline DD3X & $6.63(2)$ & $1.07(2)$ & $6.1(6)$ & $3.80(4)$ & $5.3(1)$ & $13(1)$ & & & \\
\hline DD4X & $8.30(4)$ & $1.30(4)$ & $4.9(5)$ & $4.78(5)$ & $6.9(1)$ & $19(2)$ & & & \\
\hline DD6X & $11.17(4)$ & $1.27(4)$ & $5.1(5)$ & $6.41(9)$ & $9.2(1)$ & $23(2$ & & & \\
\hline DD8X & $15.23(5)$ & $2.30(4)$ & $5.3(5)$ & $8.68(2)$ & 14.1(1) & $33(3)$ & & & \\
\hline PS1X & $2.72(2)$ & $0.88(3)$ & $2.3(2)$ & $1.72(3)$ & 1.5 & $2.9(3)$ & $2.04(3)$ & 1.5 & $0.37(3)$ \\
\hline PS2X & $3.68(2)$ & $1.16(2)$ & $2.1(2)$ & $2.08(2)$ & 2.4 & $4.9(5)$ & $2.66(2)$ & 2.4 & $0.43(4)$ \\
\hline PS3X & $4.70(3)$ & $1.04(3)$ & $2.5(3)$ & $2.65(1)$ & 3.4 & $7.8(8)$ & $3.08(2)$ & 3.4 & $0.66(5)$ \\
\hline PS4X & $6.23(2)$ & $1.25(2)$ & $2.5(3)$ & $3.25(3)$ & 4.9 & $10(1)$ & $3.94(4)$ & 4.9 & $0.93(6)$ \\
\hline PS6X & $8.79(2)$ & $1.42(2)$ & $3.1(3)$ & $4.69(3)$ & 7.5 & $16(1)$ & $5.23(4)$ & 7.5 & $1.48(9)$ \\
\hline PS8X & $10.9(1)$ & $1.88(4)$ & $2.8(3)$ & $5.47(6)$ & 9.3 & $20(2)$ & $6.49(4)$ & 9.3 & $1.62(9)$ \\
\hline
\end{tabular}

${ }^{a}$ XSW-determined mean position, $z_{0}$, and width, $\sigma$, of the Gaussian distribution for Hf. ${ }^{b}$ XSW-determined mid-height, $z_{0}$, and width, $\Delta z$, of the rectangular distribution for $\mathrm{Zr}$ and $\mathrm{Zn}+\mathrm{Re} .{ }^{c} \mathrm{XRF}-$ determined atomic coverage.

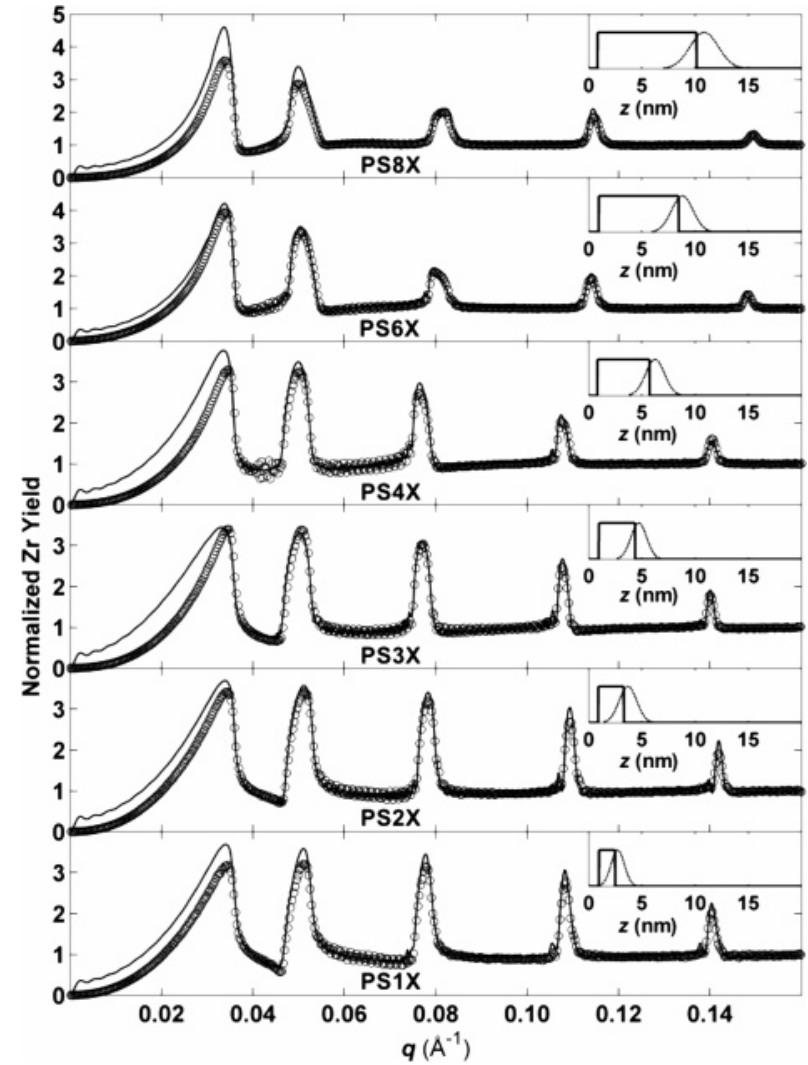

Figure 9. PSnX series XSW results showing the measured X-ray fluorescence yields for $\mathrm{Zr}$ (open circles) and $\chi^{2}$ fits (solid lines). For each sample, the inset shows the atomic distribution used for $\mathrm{Zr}$ (solid line) along with the atomic distribution used for Hf (dashed line). The atomic distributions are scaled vertically for clarity and are not proportional to the atomic coverages.

The PSnX series is based on the porphyrin square molecule whose $\mathrm{PO}_{3}$-to- $\mathrm{PO}_{3}$ axial length allows a maximum metal-tometal layer spacing of $2.5 \mathrm{~nm}$ as compared to 2.1 for the DDBPA in the DDnX series. In Figure 10b, we plot the Hf mean position along with the top, middle, and bottom of the rectangular profiles for both the $\mathrm{Zr}$ and $\mathrm{Zn}+\mathrm{Re}$ atoms. The $\chi^{2}$ fit of the Hf mean position gives $z_{0, \mathrm{Hf}}=1.20 \mathrm{~N}+1.36(\mathrm{~nm})$. The 1.20-nm per-layer slope is considerably lower than the maximum value of $2.5 \mathrm{~nm}$ permitted by the porphyrin square in a perpendicular conformation and is lower than what we observed in similar films in our previous study. ${ }^{19}$ This indicates a large tilting of the walls of the porphyrin square. The deviation of the Hf mean position for any given sample in the PS $n \mathrm{X}$ series is considerably less than in the $\mathrm{DD} n \mathrm{X}$ series indicating that this

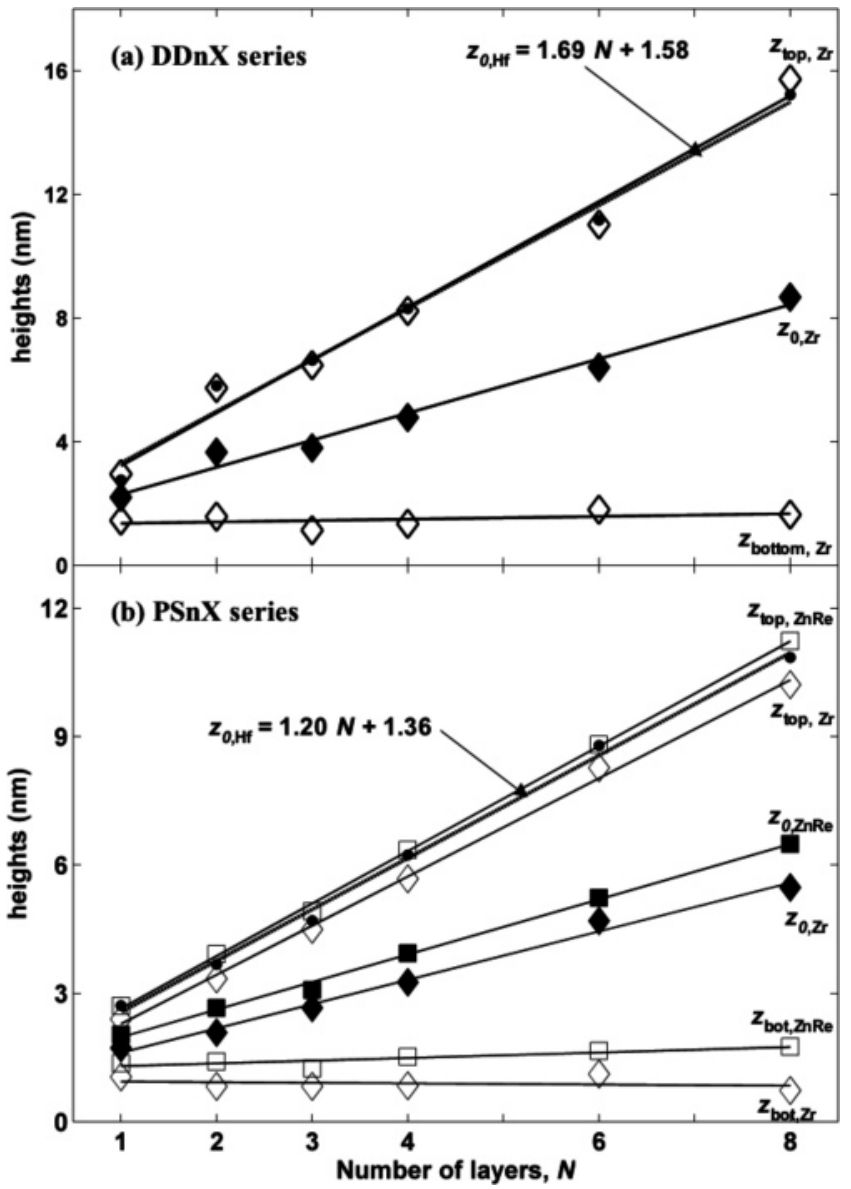

Figure 10. Atomic heights for the (a) DDnX and (b) PSnX series films. The rectangular profiles for $\mathrm{Zr}$ and $\mathrm{Zn} / \mathrm{Re}$ are shown by plotting the mid-height (filled diamonds for $\mathrm{Zr}$, filled squares for $\mathrm{Zn}+\mathrm{Re}$ ), top, and bottom (open diamonds for $\mathrm{Zr}$ and open squares for $\mathrm{Zn}+$ $\mathrm{Re})$. The Gaussian profile for Hf is shown by plotting the mean position, $z_{0}$ (closed circles). The $\chi^{2}$ fits for all are shown (solid lines).

seems to be a preferred conformation for the porphyrin square. In Figure 10b, the mid-height of the $\mathrm{Zr}$ and $\mathrm{Zn}+\mathrm{Re}$ is given by the filled diamonds and filled squares, respectively. The $\mathrm{Zn}$ $+\mathrm{Re}$ mid-height is vertically offset from the $\mathrm{Zr}$ mid-height by an average value of $0.59 \mathrm{~nm}$ which is half of the per layer spacing of $1.2 \mathrm{~nm}$. Our analysis shows that we can measure the offset between the rectangular distributions of $\mathrm{Zr}$ and $\mathrm{Zn}+$ Re in each sample in the series. This is a powerful confirmation that the ordering of $\mathrm{Zr}, \mathrm{Zn}+\mathrm{Re}$, and $\mathrm{Hf}$ in the PSnX series is precisely as we expect and is an excellent demonstration of the XSW method. 
TABLE 2: XRR and XRF Results for the DDnR and PSnR Series Films

\begin{tabular}{|c|c|c|c|c|c|c|c|c|}
\hline sample & $t_{\mathrm{F}}^{a}(\mathrm{~nm})$ & $\left(\rho_{\mathrm{F}} / \rho_{\mathrm{Si}}\right)_{\text {meas }}$ & $\sigma_{\text {int }}(\mathrm{nm})$ & $\sigma_{\text {surf }}(\mathrm{nm})$ & $\Theta_{\mathrm{Hf}^{b}}^{b}\left(\mathrm{~nm}^{-2}\right)$ & $\Theta_{\mathrm{Re}}\left(\mathrm{nm}^{-2}\right)$ & $\Theta_{\mathrm{Zr}}\left(\mathrm{nm}^{-2}\right)$ & $\left(\rho_{\mathrm{F}} / \rho_{\mathrm{Si}}\right)_{\mathrm{calc}}{ }^{c}$ \\
\hline DD1R & $3.74(4)$ & $0.75(2)$ & $0.28(5)$ & $0.77(3)$ & $5.1(5)$ & & $3.9(4)$ & \\
\hline DD2R & $5.6(1)$ & $0.72(3)$ & $0.34(1)$ & $0.49(3)$ & $4.6(5)$ & & $8.3(8)$ & \\
\hline DD3R & $7.6(2)$ & $0.72(3)$ & $0.45(4)$ & $0.97(6)$ & 4.3(5) & & $11(1)$ & \\
\hline DD4R & $9.2(1)$ & $0.71(1)$ & $0.40(4)$ & $0.86(2)$ & $5.9(6)$ & & $15(2)$ & \\
\hline DD6R & $11.9(2)$ & $0.74(1)$ & $0.40(4)$ & $1.02(4)$ & 4.6(5) & & $21(2)$ & \\
\hline DD8R & $14.6(2)$ & $0.72(1)$ & $0.40(4)$ & 1.4(1) & $4.7(5)$ & & $30(3)$ & \\
\hline PS1R & $4.39(3)$ & $0.72(1)$ & $0.1(1)$ & $0.83(2)$ & $4.2(5)$ & $0.12(2)$ & $4.0(4)$ & 0.24 \\
\hline PS2R & $4.7(1)$ & $0.75(1)$ & $0.05(5)$ & $0.98(2)$ & $3.3(4)$ & $0.39(7)$ & $7.6(8)$ & 0.43 \\
\hline PS3R & $5.8(1)$ & $0.68(3)$ & $0.12(8)$ & $0.78(1)$ & $3.4(4)$ & $0.18(3)$ & $10(1)$ & 0.26 \\
\hline PS4R & $6.1(1)$ & $0.71(4)$ & $0.3(1)$ & 1.1(1) & $3.3(4)$ & $0.24(4)$ & $10(1)$ & 0.28 \\
\hline PS6R & $9.1(1)$ & $0.70(1)$ & $0.4(1)$ & $1.1(4)$ & $3.8(4)$ & $0.34(6)$ & $18(2)$ & 0.28 \\
\hline PS8R & $12.3(2)$ & $0.61(3)$ & $0.5(1)$ & $1.6(2)$ & 4.4(5) & $0.48(9)$ & $24(2)$ & 0.28 \\
\hline
\end{tabular}

${ }^{a}$ XRR model parameters; film thickness, $t_{\mathrm{F}}$, electron density ratio, $\left(\rho_{\mathrm{F}} / \rho_{\mathrm{Si}}\right)$, film/substrate interface roughness, $\sigma_{\text {int }}$, film surface roughness, $\sigma_{\text {surf }}$. ${ }^{b} \mathrm{XRF}$ determined atomic coverage. ${ }^{c}$ Electron density ratio calculated based on measured atomic coverage.

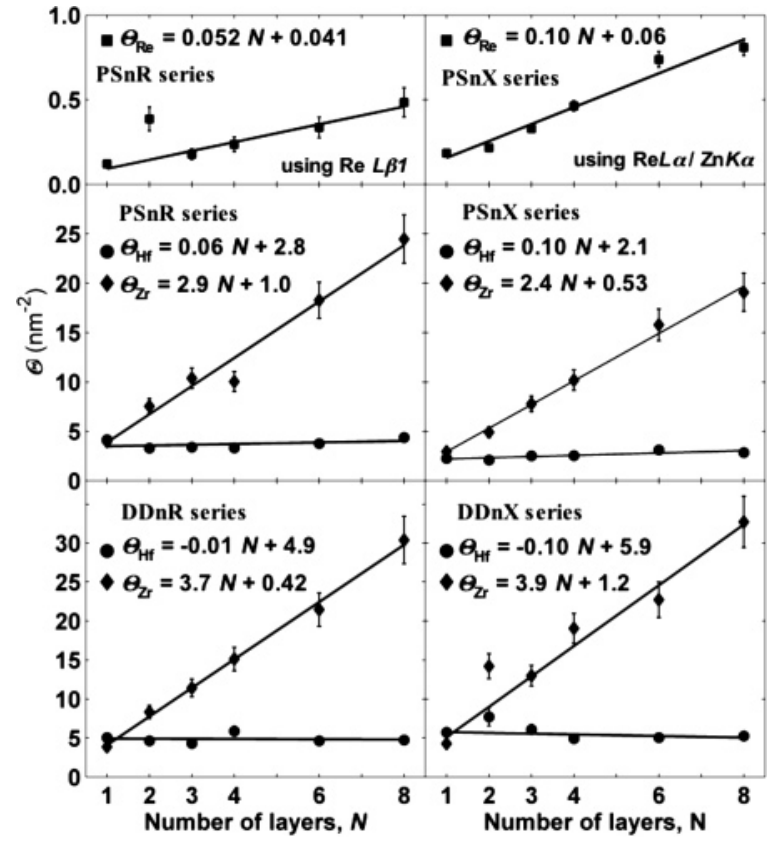

Figure 11. Plots of the measured atomic coverage of Hf (filled circles), $\mathrm{Zr}$ (filled diamonds), and $\mathrm{Zn}+\mathrm{Re}$ or $\mathrm{Re}$ (filled squares) and $\chi^{2}$ fits (solid lines).

In Figure 11, we have plotted the measured atomic coverage for the all of the samples in this study. The Hf coverage is essentially constant in any given series and is very close to the $\mathrm{Zr}$ coverage in the one layer samples. The average value of the Hf coverage in the DDnR and DD $n X$ series is 4.9 and $5.4 \mathrm{~nm}^{-2}$, respectively, whereas in the $\mathrm{PS} n \mathrm{R}$ and $\mathrm{PS} n \mathrm{X}$ series, it is 3.7 and $2.6 \mathrm{~nm}^{-2}$, respectively. The greater capacity of the DDBPA based films compared to PSBPA films is expected since the larger molecular footprint of the PSBPA will have a much lower $\mathrm{PO}_{3}$ surface density than the DDBPA. Since we have both $\mathrm{Zn}$ and Re atoms on the PSBPA molecules, the $\mathrm{Zn}$ or Re coverage gives us a measure of the coverage of the PSBPA. From Figure 11 , we see from the slope of the $\chi^{2}$ fit of the measured coverage that the per-layer coverage of PSBPA is $0.10 \mathrm{~nm}^{-2} /$ layer which in turn gives an average area per porphyrin square molecule of $40 \mathrm{~nm}^{2}$ (given $4 \mathrm{Zn}$ or Re per square). Each molecule would thus occupy a $6.3 \mathrm{~nm} \times 6.3 \mathrm{~nm}$ area. From the measured per layer thickness of $1.20 \mathrm{~nm}$, we can calculate a tilt angle of $60^{\circ}$ (where we have assumed a $2.5 \mathrm{~nm}$ for the $\mathrm{Zr}$-to- $\mathrm{Zr}$ distance based in Figure 1). The projected width of the tilted porphyrin square molecule would then be a $2.4+2.5 \sin \left(60^{\circ}\right)=4.6 \mathrm{~nm}$ assuming $2.4 \mathrm{~nm}$ is the distance between opposing zinc centers (i.e., neglecting any inward or outward bowing of the pyridyl(ethynyl)porphyrins and neglecting the van der Waals thick-

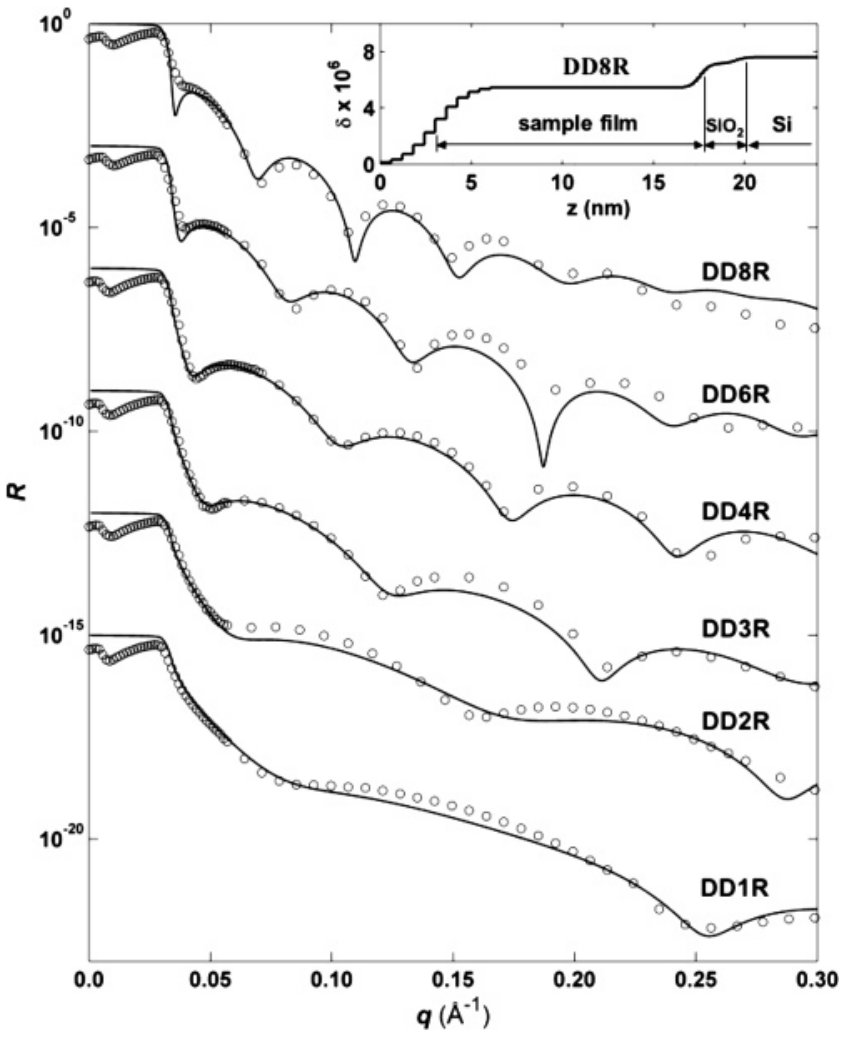

Figure 12. Typical measured (open circles) and calculated (solid lines) XRR results for the DD $n \mathrm{R}$ series samples. The inset shows the electron density profile of the layered model by plotting the real part of the index of refraction, $\delta$.

nesses of the porphyrin planes) (see Figure 1). Thus, the measured coverage agrees reasonably well with the footprint implied by the measured per-layer thickness. In a previous study, we measured an identically prepared film as PS8X (see sample D8 in ref 19) and found that the per-layer thickness was $1.61 \mathrm{~nm}$ and area per porphyrin square molecule was 12 $\mathrm{nm}^{2}$ which is a substantially different surface density but reasonably consistent with the measured tilt angle of the porphyrin square. From Table 1, we see that the $\mathrm{Zr}$ or $\mathrm{Hf}$ coverage is nearly 30 times greater than the $\mathrm{Zn}$ or Re coverage implying that there must be alternate modes of incorporation in these films. One possible mode is an extended 2D sheet of $\mathrm{ZrO}_{2}$ or $\mathrm{HfO}_{2}$ where a fraction of the $\mathrm{O}$ atoms are from the phosphonate groups and the remainder comes from the water in the solution used for the deposition of the Hf or Zr. Since there is no heavy atom in the DDBPA molecule, we were not able to measure the coverage of this molecule with our present methods. 


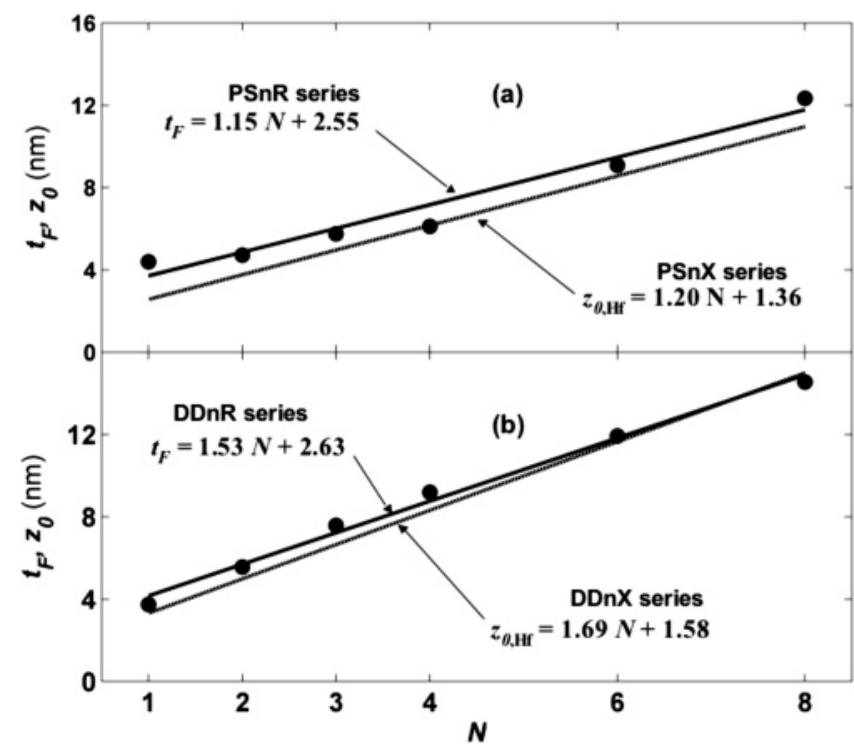

Figure 13. Results of $X R R$ analysis for (a) $D D n R$ series and (b) PSnR series. Film thicknesses (closed circles), $t_{\mathrm{F}}$, and $\chi^{2}$ fits (solid lines). Also shown for comparison are the $\chi^{2}$ fits (dashed line) of the Hf mean position, $z_{0}$, from the companion $\mathrm{DD} n \mathrm{X}$ and $\mathrm{PS} n \mathrm{X}$ series.

In Table 2, we give the results of the XRR analysis of the $\mathrm{DD} n \mathrm{R}$ and $\mathrm{PS} n \mathrm{R}$ series samples. In Figure 13, we plot the XRR measured and $\chi^{2}$ fit film thickness along with the $\chi^{2}$ fit of the Hf mean position for the XSW series. We see that the XRR determined film thickness tends to be slightly larger than the XSW measured Hf mean position. This is to be expected since the XRR method is sensitive to the maximum in the electron density gradient, which does not coincide with the Hf mean position. We can estimate the location of the maximum of the electron density gradient from the Hf atomic profile to be at $z_{0}$ $+\sigma$, which falls close to the XRR measured film height. The per-layer increases in film thickness indicated by the slopes of the Hf mean position and XRR determined film heights are in general agreement but it is important to note that the measurements come from separate sample series using different initial surfaces, i.e., sputter deposited Si for the XSW substrates versus single crystal $\mathrm{Si}$ for the XRR series. The XRR determined surface roughness in Table 2 follows the same trend as the width of the Gaussian profiles for the Hf, whereas the interface roughness is consistently in the $0.2-0.5 \mathrm{~nm}$ range for the initial substrate roughness. For both the XRR and XSW series, the very rapid roughening of the surface implies a large variability in tilt conformation of the phosphonate components.

Even though we did not observe Bragg diffraction peaks in our XRR measurements of the films (Figure 12), we still can conclude from our XSW analysis that our films are layered along the surface normal direction. Namely, the XSW determined atomic distribution profiles $\rho(\mathrm{z})$ that are averaged over a few $\mathrm{mm}^{2}$ lateral area of the X-ray footprint show that (a) in the onelayer samples DD1X and PS1X, the Zr and Hf have spatially separated distributions, (b) each sample in the DDnX and PSnX series has a Hf distribution that appears on top of the Zr slab, and (c) in the PSnX series, the $\mathrm{Zn} / \mathrm{Re}$ set of atomic layers are clearly offset from the $\mathrm{Zr}$ set of layers.

The XRR analysis also provides the overall electron density of films, which is given in Table 2 . The average electron density is $0.73 \rho_{\mathrm{Si}}$ and $0.69 \rho_{\mathrm{Si}}$ for the DDnR and PSnR series, respectively. The slightly lower electron density for the PSnR series is consistent with the more porous structure expected for these samples but far higher than expected based on the measured atomic coverage of $\mathrm{Zr}$, Hf, and $\mathrm{Zn}+\mathrm{Re}$ in the PSnX series. In
Table 2, we show the relative electron density of the film calculated according to $\left(\rho_{\mathrm{F}} / \rho_{\mathrm{Si}}\right)_{\mathrm{calc}}=\left(72 \Theta_{\mathrm{Hf}}+40 \Theta_{\mathrm{Zr}}+\right.$ $\left.2284 \Theta_{\mathrm{PS}}\right) / 699 / t_{\mathrm{F}}(72,40$, and 2284 are the number of electrons in the $\mathrm{Hf}, \mathrm{Zr}$, and porphyrin square components, respectively, and $\rho_{\mathrm{Si}}=699 \mathrm{~nm}^{-3}$ is the electron density of $\mathrm{Si}$ ). The value of the electron density based on the measured atomic coverage is only one-third of that measured by XRR. This is consistent with our previous finding ${ }^{19}$ that there must be additional material such as solvent molecules present in these films. We cannot comment on an excess electron density in the case of the DDBPA series since we do not have a measure of the coverage for this component.

\section{Conclusions}

In this study, we have shown that long-period XSW is a very useful probe of the structure of layer-by-layer assembled metal phosphonate films, providing a simultaneous measurement from multiple atomic distribution profiles of various shapes and sizes over length scales of $1-20 \mathrm{~nm}$. When combined with XRF and $\mathrm{XRR}$, we have the ability to directly evaluate important assumptions about film chemistry and quality. In the layer series based on 1,12-dodecanediylbis(phosphonic acid), we found that the per-layer thickness agrees with previously published values and observed metal layer atom densities of $\sim 5 \mathrm{~nm}^{-2}$. The Hf metal atom distributions showed that the surface roughness increases rapidly with layer number and individual samples showed large variations from the general trends but stayed within the constraints imposed by molecule length or maximum layer spacing. In the porphyrin square layer series, we observed a $1.2 \mathrm{~nm}$ per layer spacing indicating a $60^{\circ}$ tilt angle of the walls of the porphyrin square away from the surface normal. The coverage of porphyrin square molecules was found to be consistent with the area occupied by the tilted square. The layer density of $\mathrm{Hf}$ and $\mathrm{Zr}$ in the porphyrin square layer series was $\sim 30$ times higher than of the $\mathrm{PO}_{3}$ group density so that the primary mode of incorporation of the metals $\mathrm{Hf}$ and $\mathrm{Zr}$ is one other than attachment to the $\mathrm{PO}_{3}$ groups and is probably a 2-d sheet incorporating the $\mathrm{O}$ atoms of the $\mathrm{PO}_{3}$ groups as anchor points. The XRR measured film thickness was found to be consistent with the XSW determined film thickness, but the XRR measured electron density indicated the presence of additional components in the films not accounted for by the deposited metal or organophosphate components. The large $d$ spacing LSM X-ray mirrors used in this study have been shown to be excellent probes of nanoscale structure.

Acknowledgment. The authors thank Chian Liu and Raymond Conley of Argonne National Laboratory (ANL) for providing LSM substrates. The authors also thank the ESRF staff, in particular Jorg Zegenhagen, Cedric Cohen, and Lionel Andre for providing technical support. This work was supported by the National Science Foundation (NSF) under Contract EEC0118025 to the Nanoscale Science and Engineering Center at NU, the National Institutes of Health (Contract GM62109-02), the Department of Energy (DOE) under Contracts DE-FG0201ER15244 and DE-FG02-03ER15457 to the Institute for Environmental Catalysis at NU, the Camille and Henry Dreyfus Postdoctoral Program in Environmental Chemistry (fellowship for R.W.G.), and the NU Institute for BioNanotechnology in Medicine. This work made use of the NU Central Facilities supported by the MRSEC program of the NSF (DMR-0076097) and the APS X-ray Optics Fabrication Facilities at ANL supported by DOE-BES (W-31-109-Eng-38). 


\section{References and Notes} 8950.

(1) Williams, M. E.; Hupp, J. T. J. Phys. Chem. B 2001, 105, 8944-

(2) Zwahlen, M.; Tosatti, S.; Textor, M.; Hahner, G. Langmuir 2002 $18,3957-3962$

(3) Czaplewski, K. F.; Hupp, J. T.; Snurr, R. Q. Adv. Mater. 2001, 13 , 1895-1897.

(4) Zhang, J. L.; Williams, M. E.; Keefe, M. H.; Morris, G. A.; Nguyen, S. T.; Hupp, J. T. Electrochem. Solid State Lett. 2002, 5, E25-E28.

(5) Merlau, M. L.; Mejia, M. D. P.; Nguyen, S. T.; Hupp, J. T. Angew. Chem.-Int. Ed. 2001, 40, 4239-4242.

(6) Mines, G. A.; Tzeng, B. C.; Stevenson, K. J.; Li, J. L.; Hupp, J. T. Angew. Chem.-Int. Ed. 2002, 41, 154-157.

(7) Keefe, M. H.; Benkstein, K. D.; Hupp, J. T. Coord. Chem. Rev. 2000, 205, 201-228

(8) Yan, S. G.; Prieskorn, J. S.; Kim, Y. J.; Hupp, J. T. J. Phys. Chem. B 2000, 104, 10871-10877.

(9) Clearfield, A.; Wang, Z. K. J. Chem. Soc., Dalton Trans. 2002, 2937-2947.

(10) Milic, T.; Garno, J. C.; Batteas, J. D.; Smeureanu, G.; Drain, C. M. Langmuir 2004, 20, 3974-3983.

(11) Putvinski, T. M.; Schilling, M. L.; Katz, H. E.; Chidsey, C. E. D.; Mujsce, A. M.; Emerson, A. B. Langmuir 1990, 6, 1567-1571.

(12) Schilling, M. L.; Katz, H. E.; Stein, S. M.; Shane, S. F.; Wilson, W. L.; Buratto, S.; Ungashe, S. B.; Taylor, G. N.; Putvinski, T. M.; Chidsey, C. E. D. Langmuir 1993, 9, 2156-2160.

(13) Zeppenfeld, A. C.; Fiddler, S. L.; Ham, W. K.; Klopfenstein, B. J.; Page, C. J. J. Am. Chem. Soc. 1994, 116, 9158-9165.
(14) Byrd, H.; Pike, J. K.; Talham, D. R. Chem. Mater. 1993, 5, 709-

(15) Bedzyk, M. J.; Bommarito, G. M.; Schildkraut, J. S. Phys. Rev. Lett. 1989, 62, 1376-1379.

(16) Wang, J.; Bedzyk, M. J.; Penner, T. L.; Caffrey, M. Nature 1991. 354, 377-380.

(17) Bedzyk, M. J.; Bilderback, D.; White, J.; Abruna, H. D.; Bommarito, M. G. J. Phys. Chem. 1986, 90, 4926-4928.

(18) Bedzyk, M. J.; Bilderback, D. H.; Bommarito, G. M.; Caffrey, M.; Schildkraut, J. S. Science 1988, 241, 1788-1791.

(19) Libera, J. A.; Gurney, R. W.; Nguyen, S. T.; Hupp, J. T.; Liu, C.; Conley, R.; Bedzyk, M. J. Langmuir 2004, 20, 8022-8029. 6344

(20) Horne, J. C.; Blanchard, G. J. J. Am. Chem. Soc. 1998, 120, 6336-

(21) Gurney, R. W.; Massar, A. M.; Libera, J. A.; Schwartz, C. P.; Paoprasert, P.; Bailey, R. C.; Jin, H.; Morris, K. F.; Wightman, M. D.; Bedzyk, M. J.; Nguyen, S. T.; Hupp, J. T. unpublished observation.

(22) Bedzyk, M. J.; Bommarito, G. M.; Caffrey, M.; Penner, T. L. Science 1990, 248, 52-56.

(23) Puri, S.; Chand, B.; Mehta, D.; Garg, M. L.; Singh, N.; Trehan, P. N. At. Data Nucl. Data Tables 1995, 61, 289-311.

(24) Parratt, L. G. Phys. Rev. 1954, 95, 359-369.

(25) Bommarito, G. M. Ph.D. Thesis, Cornell University, Ithaca, NY, 1992.

(26) Ghose, S. K.; Dev, B. N. Phys. Rev. B 2001, 63, 245409-1-11. 